\title{
Understanding the complexities of digital dentistry integration in high-volume dental institutions
}

\author{
Leila Jahangiri, ${ }^{1}$ Guy Akiva, ${ }^{2}$ Samantha Lakhia ${ }^{3}$ and Ilser Turkyilmaz ${ }^{* 4}$
}

\section{Key points}

Digital dentistry integration in high-volume dental institutions is a complex process.
Integrated digital workflow is needed for seamless data transfer between acquisition, storage, planning and fabrication.
Safe digital data transfer in accordance with patient privacy and protection regulations set forth by governing agencies is crucial.

\begin{abstract}
The purpose of this article is to detail the primary challenges faced by large dental institutions as they incorporate digital dentistry into their mainstream workflow. Integration of digital technology is easier in private practices with smaller patient volumes and fewer trained staff required. Additionally, in private practices, scanning, designing and milling frequently occur in a single location, which does not require an external digital data transfer. However, large dental institutions must overcome several barriers which are uniquely generated by their large-scale operation. Numerous individuals must be comprehensively and efficiently trained to operate the advanced technologies. The digital software must seamlessly integrate with existing software and an internal infrastructure must be established capable of handling massive data inputs. High-volume production in large dental institutions requires the involvement of external laboratories to meet demand. This outsourcing presents a new challenge of safe digital data transfer in accordance with patient privacy and protection regulations set forth by governing agencies. It is vital for large dental institutions to recognise the unique challenges thrust upon them as they attempt to incorporate a digital workflow. With proper forethought and planning an appropriate infrastructure may be established allowing for a smooth and safe transition to the digital era.
\end{abstract}

\section{Introduction}

Digital dentistry has revolutionised the everyday operations and capabilities of dental practices. This transformation is within virtually every step of the dental workflow from treatment planning to completion of patient treatment. ${ }^{1,2,3,4}$ Digital dentistry is currently being used in many areas of dentistry, including (but not limited to) electronic health record management, digital radiography, photography, intraoral imaging, computer-aided implant planning, caries diagnosis, shade matching and occlusion analysis. ${ }^{2}$ Intraoral optical scanners (IOSs)

${ }^{1}$ Clinical Professor and Chair, New York University College of Dentistry, Department of Prosthodontics, New York, USA; ${ }^{2}$ Director, Information Technology Infrastructure and Systems Support, New York University College of Dentistry, New York, USA; ${ }^{3}$ Third-year dental student, New York University College of Dentistry, New York, USA; ${ }^{4}$ Clinical Associate Professor, New York University College of Dentistry, Department of Prosthodontics, New York, USA. *Correspondence to: Ilser Turkyilmaz Email address: ilserturkyilmaz@yahoo.com

Refereed Paper.

Accepted 14 April 2020

https://doi.org/10.1038/s41415-020-1928-5 are just one way in which this revolution has materialised. ${ }^{2,3,4}$ Intraoral optical impressions from IOSs enable digital three-dimensional (3D) capture of a patient's dentition. Intraoral optical impressions provide information on the spatial arrangement of the dentition, interocclusal relationships, and details on tooth surface texture and shade. ${ }^{5,6}$ These digital models enable more progressive treatment planning for surgical and restorative procedures. However, despite the tremendous opportunities that these technologies offer, the adaptation in dental schools has been slower than anticipated. Apart from learning a new technology, there are several challenges, including regulatory compliance, protection of patient information during data transfer, operator training and information technology integration. ${ }^{7,8,9,10}$

The components that must be taken into consideration when evaluating a digital dentistry platform include an IOS for image acquisition and a customised software for operation, as well as a treatment planning software. ${ }^{11}$ Technical challenges associated with implementation of digital dentistry into practice frequently arise during the integration of IOS software and digital patient management software with pre-existing electronic health record (EHR) systems. ${ }^{11,12}$ The value of digital dentistry is optimised if the integration with existing EHR systems and treatment software is seamless and the digital workflow is uninterrupted. In fact, software integration and data storage are typical challenges preventing the adoption of digital dentistry within dental institutions.

Safe data transfer from the site of acquisition to external laboratory partners is also necessary in high-volume dental institutions because the manufacturing of restorations and prosthesis for surgical planning cannot always occur within the institution. As data transfers occur, and with this new digital proliferation of data, breach of health records caused by cyber attacks and ransomware attacks on health insurers and providers is a concern. Therefore, measures must be considered at every step of data acquisition, storage and transfer. ${ }^{7,9,13}$ An infrastructure that allows data flow between software programs must be created, while still complying with data privacy and security provisions for safeguarding medical information, as required by the Health 


\section{Fig. 1 Three different workflows for digital dentistry}

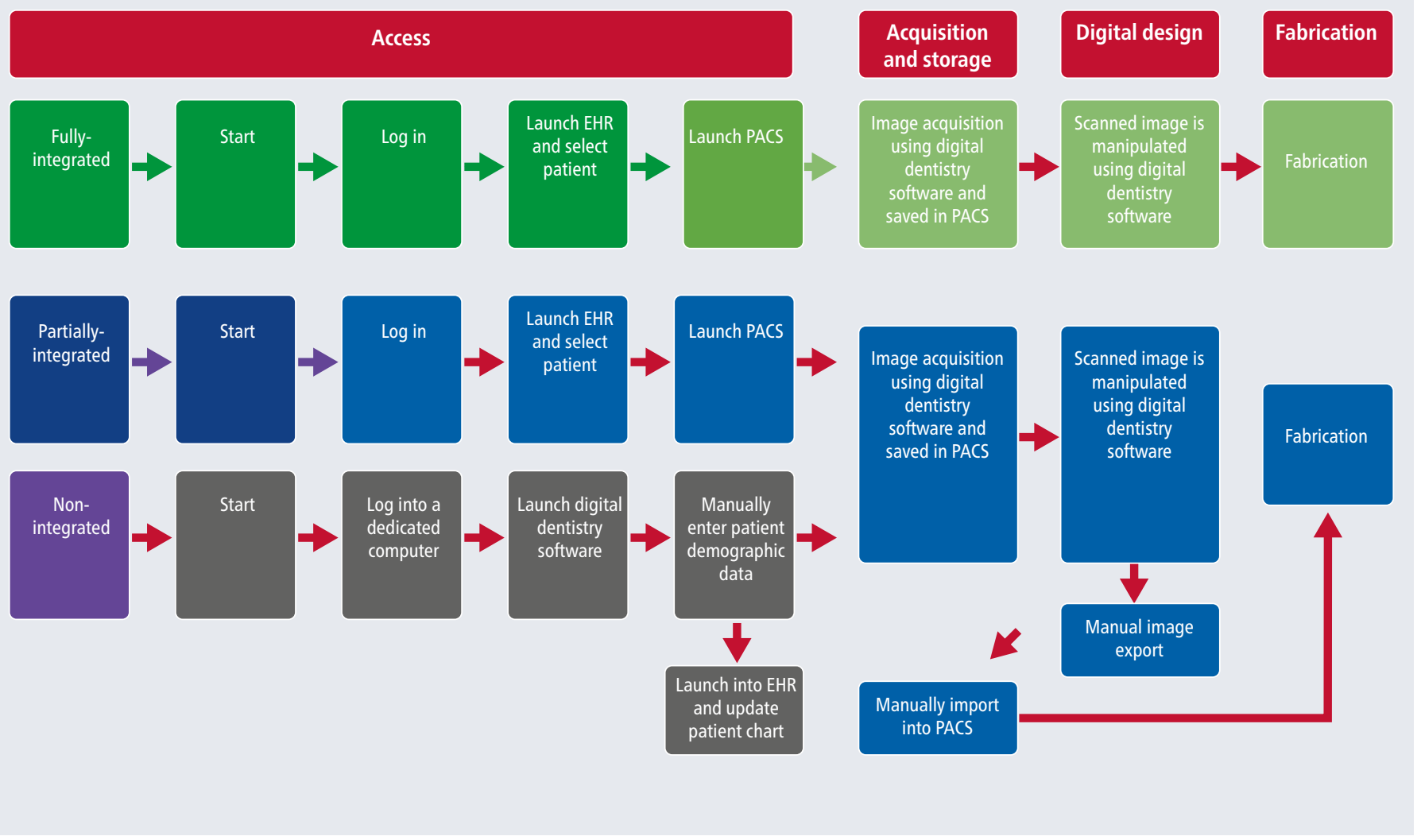

Insurance Portability and Accountability Act in the United States and the Data Protection Act in the United Kingdom. ${ }^{13,14}$ The compliance with these regulations has recently become more challenging since the digitising of medical records. ${ }^{14}$

\section{Training}

Internet-based communications and smartphones have expanded the way in which people communicate with each other and interact with the world around them. ${ }^{15}$ The expansion of digital-based communications in daily life has drastically eased the transition to, and facilitated acceptance of, electronic health records (EHR) systems within dental practices. ${ }^{16}$ Digital dentistry represents a new phase of dentistry where computer technology is an integral part of the diagnosis, planning and restorative phase, and this requires additional training. This adaptation not only requires a higher level of technology understanding, but also specific software proficiency. For optimal practice efficiency, this training goes beyond dentists and includes support staff - dental nurses, hygienists and laboratory technicians. To some extent, the continual education for all of these individuals is changing and, as we progress through this digital evolution, we will further define tasks and delegate responsibilities. There are two methods to manage training: a designated expert trainer model or a cascade effect model. ${ }^{17}$ The designated expert trainer model requires the employment of a few designated individuals who are experts in the technology to individually train all clinicians and support staff. This model typically requires a higher financial investment but allows for more uniform training of clinicians and staff. In the cascade effect model, an initial team of staff are trained by an expert and then these newly trained individuals train the next group, creating a 'train-the-trainer' effect. ${ }^{17}$ This model allows for rapid dissemination of knowledge and cost-effectiveness, but it may result in less uniform knowledge transfer. Both methods require frequent additional training and recalibration as software programs evolve and new features are introduced.

\section{Digital workflow}

A partial manual entry process is often used to create an alternative digital workflow, where data integration and transmission are interrupted. This is a detriment as it reduces efficiency, is prone to data entry mistakes and carries the potential associated risks of breach of privacy protection. Moreover, non-integrated data transfer methods may not be sufficiently adequate to handle the volume of users, data storage capacity and the transmission of patient information. For highvolume users or institutions, there has to be an assessment of the information technology (IT) infrastructure to ensure optimal usage capability. This is known as capacity management and it is to assure that the IT is of the right size and capacity for the institution's current and anticipated future needs. ${ }^{18}$

Software programs must be capable of recognising, accessing and utilising data in three primary file formats, which are used to encode information and store data following intraoral scan acquisition. ${ }^{19,20}$ These three file formats are Standard Triangle Language (STL), Object (OBJ) and Polygon (PLY). In a simple sense, these file formats are used to encode the $3 \mathrm{D}$ geometry of a model or object. STL files are translated into an 'approximate mesh', which encodes the 2D surface of an object by outlining and filling the shape of that object with adjoining triangles (facets) which meet at butt joints. STL files are most commonly utilised in $3 \mathrm{D}$ printing, but present a less 
accurate model as they do not encode any information on shade or surface texture. ${ }^{19,20}$ OBJ and PLY files are translated into a precise mesh by creating a model of an object with adjoining polygonal facets. Additionally, the two latter formats encode information on surface texture, colour and original mesh. Both approximate mesh and precise mesh models can be utilised to fabricate $3 \mathrm{D}$ objects such as dental restorations (crowns, dentures etc) by using $3 \mathrm{D}$ printers.

Although an integrated workflow is desired, an appraisal of alternative workflow options is warranted. There are three different workflows for digital dentistry: integrated, partial and non-integrated (Fig. 1).

\section{Integrated workflow}

Ideally, an integrated digital workflow utilises a seamless data transfer between acquisition, storage, planning and fabrication. This requires compatibility of components and an intermediary that allows for these applications to interact with each other. This is known as an application programing interface (API). Further, an integrated system allows for ease of access from different portals. For example, an image taken in one clinic/operatory can be viewed immediately in any other location, such as a laboratory, through a central server.

The key points of an integrated workflow are:

- Access: a provider-specific login is used to launch the practice's EHR system. Then, the provider chooses their patient and launches the practice's picture archiving and communication system (PACS)

- Acquisition: the PACS receives the acquired digital data

- Digital design: an applicable, standard digital dentistry software is used for treatment planning or design.

\section{Partially integrated workflow}

In this workflow, digital dentistry software has some integration points with existing systems, but does not integrate seamlessly into all existing software and programs. For example, demographic patient data may be integrated, while acquisition/storage and treatment planning may not.

The key points of a partially integrated workflow are:

- Access: a provider-specific login is used to launch the practice's EHR system. Then, the provider chooses their patient
- Acquisition: the provider launches proprietary digital dentistry software for scanning. After the scan is completed, the provider stores the scan in a proprietary storage system

- Digital design: the provider uses a proprietary treatment planning software and then manually exports the treatment plan to a file. Then, the provider manually imports the treatment plan to the PACS.

\section{Non-integrated workflow}

Under this workflow, the digital dentistry platform is a standalone system and does not participate in any data sharing or transmission of any kind with any existing system of record. All data are entered manually by the provider.

The key points of a non-integrated workflow are:

- Access: a provider logs into a standalone computer. The provider launches proprietary digital dentistry software and enters patient demographic data. Then, the provider logs into the EHR system (separately) and updates the patient's chart

- Acquisition: the provider launches proprietary digital dentistry software for scanning. After the scan is completed, the provider stores the scan in a proprietary storage system

- Digital design: the provider uses a proprietary treatment planning software. The provider manually exports the treatment plan to a file. Then, the provider manually imports the treatment plan to the PACS.

\section{Conclusion}

It is imperative to recognise that incorporating digital dentistry into the mainstream workflow in large dental institutions is a complex process which requires meticulous attention to detail and a carefully organised and executed infrastructure. Maintaining the integrity of patient data during acquisition, transmission and storage is paramount in our increasingly digitised existence in order to safeguard against cyber attacks and data breaches. Additionally, progressive digital technologies necessitate advanced technological training of dental staff and IT systems capable of handling massive data inputs. Advanced planning, organisation and infrastructure are critical for a seamless transition to a successful digitised workflow in high-volume dental institutions.
Conflict of interest

This research did not receive any specific grant from funding agencies in the public, commercial or not-forprofit sectors. The authors do not have any conflicts of interest for this article.

\section{References}

1. Turkyilmaz I, Unsal G S. Full-mouth rehabilitation of an elderly patient with Sjogren's syndrome by using implant-supported fixed dental prostheses including CAD/CAM frameworks. J Dent Sci 2019; 14: 428-429.

2. Blatz M B, Conejo J. The current state of chairside digita dentistry and materials. Dent Clin North Am 2019; 63: 175-197.

3. Miyoshi K, Tanaka S, Yokoyama S, Sanda M, Baba K. Effects of different types of intraoral scanners and scanning ranges on the precision of digital implant impressions in edentulous maxilla: An in vitro study. Clin Oral Implants Res 2020; 31: 74-83.

4. Takeuchi Y, Koizumi H, Furuchi M, Sato Y, Ohkubo C, Matsumura H. Use of digital impression systems with intraoral scanners for fabricating restorations and fixed dental prostheses. J Oral Sci 2018; 60: 1-7.

5. Mangano F G, Hauschild U, Veronesi G, Imburgia M, Mangano C, Admakin O. Trueness and precision of 5 intraoral scanners in the impressions of single and multiple implants: a comparative in vitro study. $B M C$ Oral Health 2019; 19: 101

6. Sadid-Zadeh R, Katsavochristou A, Squires T, Simon $M$. Accuracy of marginal fit and axial wall contour for lithium disilicate crowns fabricated using three digital workflows. J Prosthet Dent 2020; 123: 121-127.

7. Cooper L F. Digital Technology: Impact and opportunities in dental education. J Dent Educ 2019; 83: $379-380$

8. Hancocks S. What is digital about dentistry? Br Dent 2017; 223: 305

9. Sule S. Data Security, H I PAA Compliance: Steps to Prevent a Data Breach in Your Dental Practice. J Mass Dent Soc 2015; 64: 10-11.

10. Detterbeck A M, Kaiser J, Hirschfelder U. Electronic transfer of sensitive patient data. Int J Comput Dent 2015: 18: 45-57.

11. Mangano F, Gandolfi A, Luongo G, Logozzo S. Intraoral scanners in dentistry: a review of the current literature. BMC Oral Health 2017: 17: 149.

12. Afshari F S, Sukotjo C, Alfaro M F et al. Integration of Digital Dentistry into a Predoctoral Implant Program: Program Description, Rationale, and Utilization Trends. Dent Educ 2017; 81: 986-994.

13. Ramoni R B, Asher S R, White J M et al. Honouring dental patients' privacy rule right of access in the context of electronic health records. J Dent Educ 2016; 80: 691-696.

14. Scarfe W C. Data, data everywhere but not a byte to share...? Oral Surg Oral Med Oral Pathol Oral Radiol 2015; 119: 370-373.

15. Kenny P, Johnson I G. Social media use, attitudes, behaviours and perceptions of online professionalism among dental students. Br Dent J 2016; 221: 651-655.

16. Ross M K, Wei W, Ohno-Machado L. "Big data" and the electronic health record. Yearb Med Inform 2014; 9: 97-104.

17. Yarber L, Brownson C A, Jacob R R et al. Evaluating a trainthetrainer approach for improving capacity for evidence-based decision making in public health. $B M C$ Health Serv Res 2015; 15: 547.

18. Wang X, Williams C, Liu Z H, Croghan J. Big data management challenges in health research-a literature review. Brief Bioinform 2019; 20: 156-167.

19. Cevidanes L H, Ruellas A C, Jomier J et al. Incorporating 3dimensional models in online articles. Am J Orthod Dentofacial Orthop 2015; DOI: 10.1016/j. ajodo.2015.02.002.

20. Marro A, Bandukwala T, Mak W. Three-Dimensional Printing and Medical Imaging: A Review of the Methods and Applications. Curr Probl Diagn Radiol 2016; 45: 2-9. 